\title{
Prolegomena to Information Taxonomy ${ }^{\dagger}$
}

\author{
Mark Burgin ${ }^{1, *}$ and Gordana Dodig-Crnkovic ${ }^{2}$ \\ 1 Department of Mathematics, University of California, Los Angeles, 520 Portola Plaza, Los Angeles, \\ CA 90095, USA \\ 2 Department of Applied IT, Division of Cognitive Science and Communication, University of Technology \\ and University of Gothenburg, 41296 Gothenburg, Sweden; gordana.dodig-crnkovic@chalmers.se \\ * Correspondence: mburgin@math.ucla.edu \\ + Presented at the IS4SI 2017 Summit DIGITALISATION FOR A SUSTAINABLE SOCIETY, Gothenburg, \\ Sweden, 12-16 June 2017.
}

Published: 9 June 2017

Abstract: We present a part of the multiscale taxonomy of information constructed by the authors. This taxonomy is a unified system of aspect taxonomies of information.

Keywords: information; taxonomy; classification; world; law; science; brain; emotions; will

\section{Introduction}

There is a diversity of different types and kinds of information. To organize this huge collection into a system, it is necessary to classify information with respect to various criteria developing a multiscale (multiaspect) information taxonomy, in which each dimension is an aspect information taxonomy. We construct such a multiscale (multiaspect) information taxonomy based on the general theory of information [1-3] and making use of its principles and technical tools.

It is important to understand that taxonomies are not auxiliary edifices in science but they are also laws of science when scientifically grounded and validated. For instance, the biological taxonomy of Carolous Linnaeus is a law of biology in the same way as Newton's laws are laws of physics.

Here we follow taxonomic traditions of Linnaeus Carolous Linnaeus [4] and Charles Saunders Peirce [5] in the direction of information science. On the one hand, the results of our research connect new information science and technology with classical science demonstrating intrinsic links between information theory and profound results of Linnaeus. On the other hand, these results show unity in achievements of scientists working in different countries and on different continents such as biological classification of Linnaeus, chemical classification of Mendeleev, semiotic classifications of Peirce, classifications of subatomic particles in contemporary physics and classifications in information science developed here. We begin with a brief exposition of methodological principles of taxonomy construction and then apply these principles to the development of basic information taxonomies. Here we describe only some of them due to the space restrictions.

\section{Principles of Taxonomy Construction}

Having a multiplicity of objects, it is necessary to induce organization because it can help to study, understand and utilize this multiplicity. Organization is achieved by structuration of the multiplicity. An efficient technique of structuration is construction of taxonomies, classifications, typologies and categorizations. Let us consider the process and basic principles of taxonomy construction.

Taking a multiplicity of objects $M$, a researcher explicates objects' properties molding aspects or amalgamated features of $M$. Then the researcher elucidates a criterion for each aspect. This allows us to form a scale for measuring/evaluating each aspect. Such a scale together with the corresponding 
criterion allows the researcher to build an aspect taxonomy. Combining together all aspect taxonomies, the researcher obtains a multiscale taxonomy of the multiplicity $M$.

It is important to understand that according to the contemporary methodology of science, there are three types of scientific laws: classificational, equational and implicational laws. Scientists traditionally consider only two latter types as laws of nature although the first type also reflects important regularities in nature and society.

An equational law has the form of an equation, for example, of a differential equation as many laws in physics, e.g., $E=m c^{2}$, chemistry or economics.

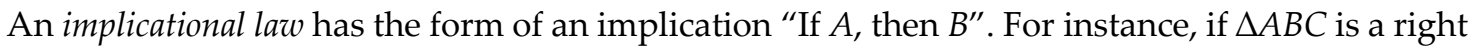
triangle, then its sides satisfy the equation $c^{2}=a^{2}+b^{2}$. It is a mathematical law called the Pythagorean theorem.

A classificational law has the form of a classification, typology or taxonomy. The biological taxonomy of the great biologist Linnaeus Carolous Linnaeus (1707-1778) and triadic typologies of the great logician Charles Saunders Peirce (1839-1914) are examples of classificational laws.

In addition, scientific laws can be qualitative and quantitative.

A quantitative law describes relations between quantitative characteristics of definite phenomena. For instance, Newton's law of motion $m a=F$ is a quantitative law of physics.

A qualitative law describes relations between qualitative characteristics of definite phenomena. For instance, Galilean law of motion "Everybody continues its state of rest or of uniform motion in a straight line unless it is compelled to change that state by forces impressed upon it" is a qualitative law of physics. Classificational laws are usually also qualitative laws.

These methodological findings determine a higher scientific status and importance of the groundbreaking Linnaeus' classification, as well as of the taxonomies constructed in this paper. Namely, this new understanding of scientific laws shows these taxonomies are qualitative laws of information science.

\section{Three Basic Taxonomies of Information}

We begin with the uppermost level of the taxonomic arrangement, which includes a huge diversity of types, kinds, sorts, categories and classes of information. On this level, we build the existential taxonomy

As information is an omnipresent phenomenon [6], it is crucial to start its classification on the global level of the whole world. This thesis implies the conjecture that the structure of the world affects existence of specific forms of information, which correspond to this structure. The large-scale structure of the world is represented by the Existential Triad of the World [7].

- $\quad$ Physical World

- Mental World

- World of Structures

In the Existential Triad, the Physical (material) World is conceived as the physical reality studied by natural sciences, the Mental World encompasses different levels of mentality, and the World of Structures consists of various forms and types of structures.

The existential stratification of the World continues the tradition of Plato with his World of Ideas [8] and the tradition of Charles Sanders Peirce with his extensive triadic classifications [5].

This stratification brings us to the phenomenon studied by the general theory of information and called information in a broad sense [3]. According to this approach, information in a broad sense is represented in each of the three worlds. In the Physical (material) World, it is called energy supporting in such a way the conjecture of von Weizsäcker that energy might in the end turn out to be information [9]. Situated at the first level of the Mental World, individual mental energy includes psychic energy studied by such psychologists as Ernst Wilhelm von Brücke (1819-1892), Sigmund Freud (1856-1939) and Carl Gustav Jung (1875-1961). Information in a broad sense, which is situated in the World of Structures, is called information in a strict sense. 


\subsection{The Global Existential Taxonomy}

As a result, we have three types of information in the global existential taxonomy:

- Physical-world information or energy

- Mental-world information and its particular case, mental energy

- Structural-world information or information per se defined as information in a strict sense

We will not analyze here the first two kinds of information in a broad sense as the first of them belongs to the scope of physics, while the second one is in the domain of psychology. Our concern is information in a strict sense or simply information.

\subsection{The Developmental Taxonomy}

Time has three modalities-past, present and future. Information is functioning and acting in time similar to all physical processes. Therefore, it is natural to distinguish types of information related to the modalities of time.

The developmental taxonomy is brought on by the temporal aspect of information:

- Potential information is measured and evaluated by its potential actions (potential impact) on the infological system.

- Actualized information is measured and evaluated by its present impact on the infological system, i.e., changes that occurred until now, that is, in the past, in the infological system.

- Emerging information is measured and evaluated by its actions that cause changes (transformations) going in the infological system at the time of measurement/evaluation, i.e., going on in the present.

Let us consider some examples.

Example 1. Information in a book before somebody reads it is potential.

It is possible to measure potential information by its potential to make changes in the corresponding infological system. For instance, measuring potential epistemic information, we estimate (measure) potential changes in the knowledge system [10].

Example 2. Information that already gave knowledge about something, e.g., information about observation of a positron obtained by Carl Anderson in 1932, is actualized.

It is possible to measure actual information by changes it made in the corresponding infological system. For instance, measuring actualized epistemic information, we determine (measure) changes in the knowledge system made by reception of this information [10].

Example 3. Information in a computer, which processes this information or in the head of a person who thinks about it, is emerging.

It is possible to estimate emerging information by its potential to make changes, by transformations it made in the corresponding infological system and by the rate of ongoing transformations. For instance, measuring emerging epistemic information, we estimate (measure) what changes in the knowledge system have already been made and reckon the rate of ongoing changes.

\subsection{Models of the Brain}

One more information taxonomy is based on the extended triune model of the brain elaborated in [3] as the further development of triune brain model of the Paul MacLean [11]. The main conception of this approach is existence of three levels of perception and action that are controlled by three 
corresponding centers in the human brain. These three centers together form the triune brain and have the structure of a triad.

Anatomically, it is possible to structure the brain into three principal parts: hindbrain (including the spinal cord), midbrain, and forebrain. According to the theory of MacLean, the neural basis, or framework, of the brain has three parts: the spinal cord, hindbrain, and midbrain. In addition to it, centuries of evolution have endowed people with three distinct cerebral systems. The oldest of them is called the reptilian brain or R-complex, which comes from reptiles. It programs behavior that is primarily related to instinctual actions based on ancestral learning and memories.

Through evolution, living beings have developed a second cerebral system, the limbic system, which MacLean calls the paleomammalian brain and which contains hippocampus, amygdala, hypothalamus, pituitary gland, and thalamus. This system is situated around the R-complex, is shared by humans with other mammals, and plays an important role in human emotional behavior.

The most recent development of the cerebral hierarchy is called the neomammalian brain, or the neocortex. It constitutes $85 \%$ of the whole human brain mass and receives its information from the external environment through the eyes, ears, and other organs of senses. The neocortex, which consists of two hemispheres, governs people creative and intellectual functions such as social interaction and advance planning. The left hemisphere works with symbolic information, applying step-by-step reasoning, while the right hemisphere handles images processed by massively parallel (gestalt) algorithms.

The extended triune model of the brain has the following structure representing three basic systems of the brain each of which is distributed through different anatomical parts of the brain:

- $\quad$ the System (Center) of Rational Intelligence (also called the System of Reasoning)

- the System (Center) of Emotions (or more generally, the System of Affective States)

- the System (Center) of Will and Instinct.

\subsection{The Formation/Action Taxonomy}

The extended triune model of the brain induces the following bifocal formation/action taxonomy/typology of information:

- $\quad$ Epistemic (form) or cognitive (action) information

- Instructional (form) or effective (action) information

- Emotional (form) or affective (action) information.

In this taxonomy, the first facet (term/name of each class) represents the form of the corresponding infological system, while the second facet (term/name of each class) represents action of information. It means that the first nominal attribute of the taxonomic classes characterizes formation aspects of information while the second operational attribute of the taxonomic classes characterizes procedural aspects of information.

Epistemic (cognitive) information is related to and processed by the System of Rational Intelligence or Reasoning.

Instructional (effective) information is related to and processed by the System of Will and Instinct.

Emotional (affective) information is related to and processed by the System of Affective States (Emotions).

To conclude, it is necessary to understand that here only a small part of the hierarchical multiscale (multiaspect) information taxonomy developed by the authors is represented. In essence, all described taxonomies together with other taxonomies constructed by the authors of this work form a hierarchical multiscale (multiaspect) information taxonomy, which gives a systematic picture of information.

Conflicts of Interest: The authors declare no conflict of interest. 


\section{References}

1. Burgin, M. Information: Problems, Paradoxes, and Solutions. J. Glob. Sustain. Inf. Soc. 2003, 1, 53-70.

2. Burgin, M. Data, Information, and Knowledge. Information 2004, 7, 47-57.

3. Burgin, M. Theory of Information: Fundamentality, Diversity and Unification; World Scientific: New York, NY, USA; London, UK; Singapore, 2010.

4. Linnaeus, C. Systema Naturae, Sive Regna Tria Naturae Systematice proPosita per Classes, Ordines, Genera $\mathcal{E}$ Species; Johann Wilhelm de Groot for Theodor Haak: Leiden, The Netherlands, 1735.

5. Peirce C.S. Collected Papers; Cambridge University Press: Cambridge, UK, 1931-1935; Volume 1-6.

6. Burgin, M.; Dodig-Crnkovic, G. Information and Computation-Omnipresent and Pervasive. In Information and Computation; World Scientific: New York, NY, USA; London, UK; Singapore, 2011; pp. 7-32.

7. Burgin, M. Structural Reality; Nova Science Publishers: Hauppauge, NY, USA, 2012.

8. Plato. The Collected Dialogues of Plato; Princeton University Press: Princeton, NJ, USA, 1961.

9. Von Weizsäcker, C.F. Die Einheit der Natur; Deutscher Taschenbuch Verlag: Munich, Germany, 1974

10. Burgin, M. Epistemic Information in Stratified M-Spaces. Information 2011, 2, 697-726.

11. MacLean, P.D. A Triune Concept of the Brain and Behavior; University of Toronto Press: Toronto, ON, USA, 1973.

(C) 2017 by the authors. Licensee MDPI, Basel, Switzerland. This article is an open access article distributed under the terms and conditions of the Creative Commons Attribution (CC BY) license (http://creativecommons.org/licenses/by/4.0/). 